\title{
Endpoint Estimates for Generalized Commutators of Hardy Operators on $H^{1}$ Space
}

\author{
Xiao Yu ${ }^{1,2}$ and Shanzhen $L u^{3}$ \\ ${ }^{1}$ Department of Mathematics, Shangrao Normal University, Shangrao City 334001, China \\ ${ }^{2}$ Department of Mathematics, Zhejiang University, Hangzhou City 310027, China \\ ${ }^{3}$ School of Mathematical Science, Beijing Normal University, Beijing City 100875, China \\ Correspondence should be addressed to Xiao Yu; xyuzju@163.com
}

Received 25 March 2013; Accepted 7 May 2013

Academic Editor: Dashan Fan

Copyright $\odot 2013 \mathrm{X}$. Yu and S. Lu. This is an open access article distributed under the Creative Commons Attribution License, which permits unrestricted use, distribution, and reproduction in any medium, provided the original work is properly cited.

We study the $H^{1}$-boundedness of the generalized commutators of Hardy operator with a homogeneous kernel as follows: $\mathscr{H}_{\Omega, A, \beta}^{m} f(x)=\left(1 /|x|^{n-\beta}\right) \int_{|y|<|x|}\left(\Omega(x-y) /|x-y|^{m-1}\right) R_{m}(A ; x, y) f(y) d y$, where $R_{m}(A ; x, y)=A(x)-\sum_{|\alpha|<m}(1 / \alpha !) D^{\alpha} A(y)(x-y)^{\alpha}$ with $m \in Z^{+}, 0 \leq \beta<n$ and $\Omega \in \operatorname{Lip}_{1}\left(S^{n-1}\right)$. We prove that, when $m \geq 1, \mathscr{H}_{\Omega, A, \beta}^{m}$ is not bounded from $H^{1}$ to $L^{n /(n-\beta)}$ unless $\mathscr{H}_{\Omega, A, \beta}^{m} \equiv 0$. Finally, we prove that $\mathscr{H}_{\Omega, A, \beta}^{m}$ is bounded from $H^{1}$ to $L^{n /(n-\beta), \infty}$ with $m \geq 1$.

\section{Introduction}

Let $f \in L^{p}\left(\mathbb{R}^{+}\right)$with $1<p<\infty$; the classical Hardy operator is defined by

$$
H f(x)=\frac{1}{x} \int_{0}^{x} f(t) d t, \quad x \neq 0 .
$$

A famous result proved by Hardy [1] can be stated as follows;

$$
\|H f\|_{L^{p}\left(\mathbb{R}^{+}\right)} \leq \frac{p}{p-1}\|f\|_{L^{p}\left(\mathbb{R}^{+}\right)} .
$$

Hardy [1] also pointed out the fact that the constant $p /(p-1)$ in (2) is the best possible. Later, Hardy operator was studied by many mathematicians; please see $[2,3]$ for more details.

In 1995, Christ and Grafakos [4] studied the following $n$ dimensional Hardy operator;

$$
\mathscr{H} f(x)=\frac{1}{v_{n}|x|^{n}} \int_{|y|<|x|} f(y) d y, \quad x \in \mathbb{R}^{n} \backslash\{0\},
$$

where $v_{n}$ is the volume of the unit ball in $\mathbb{R}^{n}$, and they proved the following inequality:

$$
\|\mathscr{H} f\|_{L^{p}} \leq \frac{p}{p-1}\|f\|_{L^{p}}, \quad 1<p<\infty .
$$

Furthermore, Christ and Grafakos [4] also showed that the constant $p /(p-1)$ in (3) is the best possible.

In 2007, Fu et al. [5] considered the following commutator of fractional Hardy operator:

$$
\mathscr{H}_{\beta}^{b} f(x)=b(x) \mathscr{H}_{\beta} f(x)-\mathscr{H}_{\beta}(f b)(x),
$$

where $\mathscr{H}_{\beta} f(x)$ is defined by

$$
\mathscr{H}_{\beta} f(x)=\frac{1}{|x|^{n-\beta}} \int_{|y|<|x|} f(y) d y
$$

with $-n<\beta<n$. When $\beta=0$, we simply denote $\mathscr{H}_{0}^{b}$ by $\mathscr{H}_{b}$ and $\mathscr{H}_{0}$ is just the $n$-dimensional Hardy operator proposed by Christ and Grafakos in [4] (without considering the constant $\left.v_{n}\right)$.

In 2011, Fu et al. [6] studied the following $n$-dimensional fractional Hardy operator with a homogeneous kernel:

$$
\mathscr{H}_{\Omega, \beta} f(x)=\frac{1}{|x|^{n-\beta}} \int_{|y|<|x|} \Omega(x-y) f(y) d y,
$$

where $\Omega \in L^{r}\left(S^{n-1}\right)$. Fu et al. [6] proved that $\mathscr{H}_{\Omega, \beta}^{b}$ is bounded on Herz type space and $\lambda$-central Morrey space. Here $\mathscr{H}_{\Omega, \beta}^{b}$ 
is just the commutator of fractional Hardy operator with a homogeneous kernel.

Recently, Zhao et al. [7] gave a counterexample to show that $\mathscr{H}_{b}$ is not bounded from $H^{1}$ to $L^{1}$, and they proved that $\mathscr{H}_{b}$ is bounded from $H^{1}$ to weak $L^{1}$ space where $H^{1}$ denotes the Hardy space.

On the other hand, in 1982, Cohen [8] studied the following generalized commutator:

$$
\begin{aligned}
T_{A}^{2} f(x)=\int_{\mathbb{R}^{n}} \frac{\Omega(x-y)}{|x-y|^{n+1}} \\
\\
\quad \times(A(x)-A(y)-\nabla A(y)(x-y)) f(y) d y,
\end{aligned}
$$

where $\Omega \in L^{1}\left(S^{n-1}\right)$ is homogeneous of degree zero and satisfies the moment condition

$$
\int_{S^{n-1}} \Omega(x) x^{\alpha} d \sigma(x)=0
$$

for $|\alpha|=1$. Cohen [8] proved that, if $\Omega \in \operatorname{Lip}_{1}\left(S^{n-1}\right)$ and $\nabla A \in \mathrm{BMO}$, then $T_{A}^{2}$ is bounded on $L^{p}\left(\mathbb{R}^{n}\right)$ with $1<p<\infty$.

Later, Cohen and Gosselin [9] considered another type of generalized commutator $T_{A}^{m}(f)$ as follows:

$$
T_{A}^{m} f(x)=\int_{\mathbb{R}^{n}} \frac{\Omega(x-y)}{|x-y|^{n+m-1}} R_{m}(A ; x, y) f(y) d y,
$$

where $R_{m}(A ; x, y)\left(m \in Z^{+}\right)$is defined by $R_{m}(A ; x, y)=$ $A(x)-\sum_{|\alpha|<m}(1 / \alpha !) D^{\alpha} A(y)(x-y)^{\alpha}$, the $m$ th remainder of Taylor series of the function $A$ at $x$ about $y$, and $\Omega$ satisfies the following moment conditions:

$$
\int_{S^{n-1}} \Omega(x) x^{\alpha} d \sigma(x)=0
$$

for $|\alpha|=m-1$. Obviously, if we choose $m=1, T_{A}^{m}$ becomes $[A, T]$, the commutator of $T$ generalized by $A$ and $T$.

Cohen and Gosselin [9] proved that $T_{A}^{m}$ is bounded on $L^{p}\left(\mathbb{R}^{n}\right)$ for $1<p<\infty$ if $\Omega \in \operatorname{Lip}_{1}\left(S^{n-1}\right)$ and the function $A$ has derivatives of order $m-1$ in $\operatorname{BMO}\left(\mathbb{R}^{n}\right)$. Later, $T_{A}^{m}$ was studied by many mathematicians; for example, see $[10,11]$ or [12] for more details. Particularly in [11], Lu and Wu studied the endpoint estimates of $T_{A}^{m}$ on $H^{1}$ space.

It should be pointed out that the generalized commutators of some operators play an important role in the study of partial differential equation. Recently, by using the $W^{1, p}$ estimate for the elliptic equation of divergence form with partially BMO coefficients and the $L^{p}$ boundedness of the Cohen-Gosselin type generalized commutators proved by Yan in [12], Wang and Zhang [13] gave a new proof of Wu's theorem in [14]. Here we would like to point out that the method used in [13] is much simpler than that in [14].

In this paper, we will consider the following generalized commutator of fractional Hardy operator with a homogeneous kernel:

$$
\begin{aligned}
& \mathscr{H}_{\Omega, A, \beta}^{m} f(x) \\
& =\frac{1}{|x|^{n-\beta}} \int_{|y|<|x|} \frac{\Omega(x-y)}{|x-y|^{m-1}} f(y) R_{m}(A ; x, y) d y,
\end{aligned}
$$

where $R_{m}(A ; x, y)=A(x)-\sum_{|\alpha|<m}(1 / \alpha !) D^{\alpha} A(y)(x-y)^{\alpha}, 0 \leq$ $\beta<n$, and $\Omega \in \operatorname{Lip}_{1}\left(S^{n-1}\right)$.

As the Hardy operator is controlled by the HardyLittlewood maximal function, we have

$$
\mathscr{H}_{\Omega, A, \beta}^{m} \leq C M_{\Omega, A, \beta} f(x),
$$

where $M_{\Omega, A, \beta} f(x)$ is defined by

$$
\begin{aligned}
& M_{\Omega, A, \beta} f(x) \\
& \quad=\sup _{r>0} \frac{1}{r^{n-\beta}} \int_{|x-y|<r} \frac{|\Omega(x-y)|}{|x-y|^{m-1}}\left|R_{m}(A ; x, y) f(y)\right| d y .
\end{aligned}
$$

By a simple computation or from [15, pp. 221-222], we have

$$
\begin{aligned}
& M_{\Omega, A, \beta} f(x) \\
& \quad=\sup _{r>0} \frac{1}{r^{n-\beta}} \int_{|x-y|<r} \frac{|\Omega(x-y)|}{|x-y|^{m-1}}\left|R_{m}(A ; x, y) f(y)\right| d y \\
& \quad \leq C \sup _{r>0} \frac{1}{r^{n-\beta}} \int_{(r / 2)<|x-y|<r} \frac{|\Omega(x-y)|}{|x-y|^{m-1}}\left|R_{m}(A ; x, y) f(y)\right| d y,
\end{aligned}
$$

and thus

$$
\begin{aligned}
& \mathscr{H}_{\Omega, A, \beta}^{m} f(x) \\
& \leq C \sup _{r>0} \frac{1}{r^{n-\beta}} \int_{|x-y|<r} \frac{|\Omega(x-y)|}{|x-y|^{m-1}}\left|R_{m}(A ; x, y) f(y)\right| d y \\
& \leq C \sup _{r>0} \frac{1}{r^{n-\beta}} \int_{(r / 2)<|x-y|<r} \frac{|\Omega(x-y)|}{|x-y|^{m-1}}\left|R_{m}(A ; x, y) f(y)\right| d y \\
& \leq C \sup _{r>0} r^{-(n-\beta+m-1)} \int_{(r / 2)<|x-y|<r}|\Omega(x-y)| \\
& \quad \times\left|R_{m}(A ; x, y) f(y)\right| d y .
\end{aligned}
$$

So we have

$$
\mathscr{H}_{\Omega, A, \beta}^{m} f(x) \leq C \widetilde{M}_{\Omega, \beta}^{A} f(x)
$$

where

$$
\begin{aligned}
\widetilde{M}_{\Omega, \beta}^{A} f(x)= & \sup _{r>0} r^{-(n-\beta+m-1)} \\
& \times \int_{(r / 2)<|x-y|<r}\left|\Omega(x-y) R_{m}(A ; x, y) f(y)\right| d y .
\end{aligned}
$$

From $[15$, p. 222], we have the following lemma.

Lemma 1 (see [15]). Let $0<\beta<n$ and $\Omega \in \operatorname{Lip}_{1}\left(S^{n-1}\right)$. If $1<p, q<\infty$ with $1 / p-1 / q=\beta / n$, and $A$ has derivatives of order $m-1$ in $B M O\left(\mathbb{R}^{n}\right)$, then

$$
\left\|\widetilde{M}_{\Omega, \beta}^{A} f\right\|_{L^{q}} \leq C \sum_{|\alpha|=m-1}\left\|D^{\alpha} A\right\|_{B M O}\|f\|_{L^{p}},
$$

where the constant $C$ is independent of $f$ and $A$. 
By checking [15, p. 222] carefully, we deduce that (19) still holds if we take $\beta=0$. So we have the following proposition.

Proposition 2. Let $0 \leq \beta<n, 1 / p-1 / q=\beta / n$ with $1<$ $p, q<\infty$. If $\Omega \in \operatorname{Lip}_{1}\left(S^{n-1}\right)$ and $A$ has derivatives of order $m-1$ in $B M O\left(\mathbb{R}^{n}\right)$, then

$$
\left\|\mathscr{H}_{\Omega, A, \beta}^{m} f\right\|_{L^{q}} \leq C \sum_{|\alpha|=m-1}\left\|D^{\alpha} A\right\|_{B M O}\|f\|_{L^{p}}
$$

where the constant $C$ is independent of $f$ and $A$.

Definition 3 (see [16]). One says a function $a(x)$ is an $H^{1}$ atom if $a$ satisfies the following conditions:

$$
\begin{aligned}
& \text { (i) } \operatorname{supp}(a) \subset B\left(x_{0}, r\right), \\
& \text { (ii) }\|a\|_{L^{\infty}} \leq\left|B\left(x_{0}, r\right)\right|^{-1}, \\
& \text { (iii) } \int a(x) d x=0 .
\end{aligned}
$$

It is well known that, if a function $f$ belongs to $H^{1}$, then it can be written as $f=\sum_{i=1}^{\infty} \lambda_{i} a_{i}$ where each $a_{i}$ is an $H^{1}$ atom. Moreover, one has

$$
\|f\|_{H^{1}} \sim \inf \left\{\sum_{i=-\infty}^{+\infty}\left|\lambda_{i}\right|\right\},
$$

where the infimum is taken over all decompositions of $f$.

Definition 4 (see [17]). A function $f$ is said to belong to $\mathrm{BMO}\left(\mathbb{R}^{n}\right)$ if the following sharp maximal function is bounded:

$$
f^{\sharp}(x)=\sup _{B} \frac{1}{|B|} \int_{B}\left|f(y)-f_{B}\right| d y<\infty,
$$

where the supreme is taken over all balls $B \subset \mathbb{R}^{n}$ and $f_{B}=$ $(1 /|B|) \int_{B} f(x) d x$ and $\|f\|_{\text {BMO }}=\left\|f^{\sharp}\right\|_{L^{\infty}}$.

Proposition 5 (see [17]). Let $1<p<\infty$ and $f \in B M O\left(\mathbb{R}^{n}\right)$; then one has

(a) $\|f\|_{B M O} \sim \sup _{B}\left((1 /|B|) \int_{B}\left|f(x)-f_{B}\right|^{p} d x\right)^{1 / p}$;

(b) $\|f\|_{B M O} \sim \sup _{B} \inf _{a \in R}(1 /|B|) \int_{B}|f(x)-a| d x$.

Obviously, when $m=1, \mathscr{H}_{\Omega, A, \beta}^{m}$ can be written as $\mathscr{H}_{\Omega, A, \beta}^{1} f(x):=\mathscr{H}_{\Omega, A, \beta} f(x)=A(x) \mathscr{H}_{\Omega, \beta} f(x)-\mathscr{H}_{\Omega, \beta}(f A)(x)$, just the commutator of fractional Hardy operator with a homogeneous kernel.

For the case $m=2, \beta=0$, and $\Omega \equiv 1$, Lu and Zhao [18] proved that $\mathscr{H}_{\Omega, A, 0}^{2}$ is bounded on Herz type spaces and Morrey-Herz type spaces.

In this paper, we would like to show that $\mathscr{H}_{\Omega, A, \beta}^{m}$ is not bounded from $H^{1}$ to $L^{n /(n-\beta)}$ for all $m \in Z^{+}$. Furthermore, we will prove that $\mathscr{H}_{\Omega, A, \beta}^{m}$ is bounded from $H^{1}$ to $L^{n /(n-\beta), \infty}$, where $L^{n /(n-\beta), \infty}$ denotes the weak $L^{n /(n-\beta)}$ space. Some ideas of this paper come from Zhao et al. [7].
In this chapter, we would like to show that, if $A \in$ $\operatorname{BMO}\left(\mathbb{R}^{n}\right), \mathscr{H}_{\Omega, A, \beta}(0 \leq \beta<n)$ is not bounded from $H^{1}$ to $L^{n /(n-\beta)}$.

To show this, let $A(x)=\chi_{(4, \infty)}(x) \in \mathrm{BMO}, \Omega \equiv 1$, and $f_{0}(x)=\chi_{(0,4)}(x)-\chi_{(-4,0)}(x)$; then for $x>8$ and $n=1$, we have

$$
\left|\mathscr{H}_{\Omega, A, \beta} f_{0}(x)\right|=\left|\frac{1}{x^{1-\beta}} \int_{0}^{4}(1-0) \times 1 d y\right|=\frac{4}{x^{1-\beta}},
$$

and then

$$
\int_{R^{1}}\left|\mathscr{H}_{\Omega, A, \beta} f_{0}(x)\right|^{1 /(1-\beta)} d x \geq \int_{8}^{\infty} \frac{4^{1 /(1-\beta)}}{x} d x=\infty,
$$

which indicates that $\mathscr{H}_{\Omega, A, \beta}$ is not bounded from $H^{1}$ to $L^{n /(n-\beta)}$.

\section{Endpoint Estimates for $\mathscr{H}_{\Omega, A, \beta}^{m}$ from $H^{1}$ to $L^{n /(n-\beta)}$}

In Section 1, we know that, when $m=1, \mathscr{H}_{\Omega, A, \beta}^{1}$ is not bounded from $H^{1}$ to $L^{n /(n-\beta)}$. In this section, we will prove that, when $m \geq 2, \mathscr{H}_{\Omega, A, \beta}^{m}$ is also not bounded from $H^{1}$ to $L^{n /(n-\beta)}$ unless $\mathscr{H}_{\Omega, A, \beta}^{m} \equiv 0$. We have the following conclusions.

Theorem 6. Let $m \geq 2,0 \leq \beta<n$, and $\Omega \in \operatorname{Lip}_{1}\left(S^{n-1}\right)$. Assume that $A$ has derivatives of order $m-1$ in $B M O\left(\mathbb{R}^{n}\right)$; then the following two statements are equivalent;

(i) $\mathscr{H}_{\Omega, A, \beta}^{m}$ maps $H^{1}\left(\mathbb{R}^{n}\right)$ continuously into $L^{n /(n-\beta)}$;

(ii) for any $H^{1}$ atom supported on certain ball $B$ and $u \in$ $3 B \backslash 2 B$, there is

$$
\begin{aligned}
& \int_{(4 B)^{c}}\left|\sum_{|\alpha|=m-1} \frac{1}{\alpha !} K_{\alpha}(x, u) \frac{1}{|x|^{n-\beta}} \int_{B} D^{\alpha} A(y) a(y) d y\right|^{n /(n-\beta)} \\
& \quad \times d x \leq C,
\end{aligned}
$$

where $K_{\alpha}(x, u)=\Omega(x-u)(x-u)^{\alpha} /|x-u|^{m-1}$ with $|\alpha|=m-1$.

In order to prove Theorem 6, we need the following lemma.

Lemma 7 (see [9]). Let b be a function on $\mathbb{R}^{n}$ with $m$ th order derivatives in $L_{\text {loc }}^{q}\left(\mathbb{R}^{n}\right)$ for some $q>n$; then

$$
\begin{aligned}
\left|R_{m}(b ; x, y)\right| \leq & C_{m, n}|x-y|^{m} \\
& \times \sum_{|\alpha|=m}\left(\frac{1}{|\widetilde{Q}(x, y)|} \int_{\widetilde{\mathrm{Q}}(x, y)}\left|D^{\alpha} b(z)\right|^{q} d z\right)^{1 / q},
\end{aligned}
$$

where $\widetilde{Q}(x, y)$ is the cube centered at $x$ and having diameter $5 \sqrt{n}|x-y|$. 
Proof of Theorem 6. Suppose that $a(x)$ is an $H^{1}$ atom supported on $B\left(x_{0}, r\right)$ and satisfies (21). Now we take $\widetilde{a}(x)=$ $a\left(x+x_{0}\right)$; then $\tilde{a}$ is also an $H^{1}$ atom and satisfies

$$
\begin{aligned}
& \left(\mathrm{i}^{\prime}\right) \operatorname{supp}(\widetilde{a}) \subset B(0, r), \\
& \left(\mathrm{ii}^{\prime}\right)\|\widetilde{a}\|_{L^{\infty}} \leq|B(0, r)|^{-1}, \\
& \left(\mathrm{iii}^{\prime}\right) \int_{\mathbb{R}^{n}} \widetilde{a}(x) d x=0 .
\end{aligned}
$$

Thus by the main results in [19] and the atomic decomposition of the space $H^{1}\left(\mathbb{R}^{n}\right)$, it suffices to show that, for any $H^{1}$ atom $\tilde{a}$, we have $\left\|\mathscr{H}_{\Omega, A, \beta}^{m} \tilde{a}\right\|_{L^{n /(n-\beta)}} \leq C$.

Let $B=B(0, r)$ and $\widetilde{A}(x)=A(x)-\sum_{|\alpha|=m-1}(1 /$ $\alpha !) m_{B}\left(D^{\alpha} A\right) x^{\alpha}$; then $R_{m}(A ; x, y)=R_{m}(\widetilde{A} ; x, y)$. For each $H^{1}$ atom $\tilde{a}$, we split each $\mathscr{H}_{\Omega, A, \beta}^{m} \tilde{a}(x)$ as

$$
\begin{aligned}
& \mathscr{H}_{\Omega, A, \beta}^{m} \tilde{a}(x) \\
& =\chi_{4 B}(x) \mathscr{H}_{\Omega, A, \beta}^{m} \tilde{a}(x)+\chi_{(4 B)^{c}}(x) \mathscr{H}_{\Omega, A, \beta}^{m} \tilde{a}(x) \\
& :=\mu_{1}(x)+\mu_{2}(x) .
\end{aligned}
$$

For $\mu_{1}(x)$, taking $n /(n-\beta)<q<\infty$ and $p$ so that $1 / p-$ $1 / q=\beta / n$, it follows from Proposition 2 that

$$
\begin{aligned}
\left\|\mu_{1}\right\|_{L^{n /(n-\beta)}} & \leq|4 B|^{(n-\beta) / n-1 / q}\left\|\mathscr{H}_{\Omega, A, \beta}^{m} \tilde{a}\right\|_{L^{q}} \\
& \leq C \sum_{|\alpha|=m-1}\left\|D^{\alpha} A\right\|_{\mathrm{BMO}}|B|^{1-1 / p}\|\widetilde{a}\|_{L^{p}} \\
& \leq C \sum_{|\alpha|=m-1}\left\|D^{\alpha} A\right\|_{\mathrm{BMO}} .
\end{aligned}
$$

For $\mu_{2}(x)$, as $x \in(4 B)^{c}$ and $|y|<|x|$, we can deduce $\{y:|y|<|x|\} \cap\{y: y \in B(0, r)\}=\{y: y \in B(0, r)\}$; thus we have

$$
\begin{aligned}
\mu_{2}(x)= & \chi_{(4 B)^{c}} \frac{1}{|x|^{n-\beta}} \\
& \times \int_{B(0, r)} \frac{\Omega(x-y)}{|x-y|^{m-1}} R_{m}(\widetilde{A} ; x, y) \widetilde{a}(y) d y .
\end{aligned}
$$

Next we denote $B(0, r)=B$ and $B(0, k r)=k B$; then by the vanishing condition of $\tilde{a}$, we decompose $\mu_{2}$ as follows:

$$
\begin{array}{r}
\mu_{2}(x) \\
=\chi_{(4 B)^{c}}(x) \frac{1}{|x|^{n-\beta}} \int_{B}\left(\frac{\Omega(x-y)}{|x-y|^{m-1}} R_{m-1}(\widetilde{A} ; x, y)\right. \\
\left.\quad-\frac{\Omega(x-u)}{|x-u|^{m-1}} R_{m-1}(\widetilde{A} ; x, u)\right)
\end{array}
$$

$$
\begin{aligned}
& \times \widetilde{a}(y) d y-\chi_{(4 B)^{c}}(x) \frac{1}{|x|^{n-\beta}} \\
& \times \sum_{|\alpha|=m-1} \frac{1}{\alpha !} \int_{B}\left[K_{\alpha}(x, y)-K_{\alpha}(x, u)\right] D^{\alpha} \widetilde{A}(y) \widetilde{a}(y) d y \\
& -\chi_{(4 B)^{c}}(x) \frac{1}{|x|^{n-\beta}} \\
& \times \sum_{|\alpha|=m-1} \frac{1}{\alpha !} \int_{B} K_{\alpha}(x, u) D^{\alpha} \widetilde{A}(y) \tilde{a}(y) d y \\
& =\mu_{21}(x, u)-\mu_{22}(x, u)-\mu_{23}(x, u),
\end{aligned}
$$

where $u \in 3 B \backslash 2 B$.

For $\mu_{21}(x, u)$, as $u \in 3 B \backslash 2 B, y \in B$, and $x \in(4 B)^{c}$, we have

$$
|x-y| \sim|x-u| \sim|x| .
$$

Thus we have

$$
\begin{aligned}
& \left|\frac{\Omega(x-y)}{|x-y|^{m-1}} R_{m-1}(\widetilde{A} ; x, y)-\frac{\Omega(x-u)}{|x-u|^{m-1}} R_{m-1}(\widetilde{A} ; x, u)\right| \\
& \leq\left|\frac{\Omega(x-y)}{|x-y|^{m-1}} R_{m-1}(\widetilde{A} ; x, y)-\frac{\Omega(x-y)}{|x-u|^{m-1}} R_{m-1}(\widetilde{A} ; x, y)\right| \\
& \quad+\left|\frac{\Omega(x-y)}{|x-u|^{m-1}} R_{m-1}(\widetilde{A} ; x, y)-\frac{\Omega(x-u)}{|x-u|^{m-1}} R_{m-1}(\widetilde{A} ; x, y)\right| \\
& \quad+\left|\frac{\Omega(x-u)}{|x-u|^{m-1}}\right|\left|R_{m-1}(\widetilde{A} ; x, y)-R_{m-1}(\widetilde{A} ; x, u)\right| \\
& :=I+I I+I I I .
\end{aligned}
$$

For the term $I$, by Lemma 7, we have

$$
\begin{aligned}
I & \leq C \frac{|\Omega(x-y)|}{|x-y|^{m}}|y-u|\left|R_{m-1}(\widetilde{A} ; x, y)\right| \\
& \leq C \frac{|y-u|}{|x-y|^{m}}|x-y|^{m-1} k \sum_{|\alpha|=m-1}\left\|D^{\alpha} A\right\|_{\mathrm{BMO}} \\
& \leq C k 2^{-k} \sum_{|\alpha|=m-1}\left\|D^{\alpha} A\right\|_{\mathrm{BMO}} .
\end{aligned}
$$

For the term $I I$, by the similar estimates of $I$, we obtain

$$
I I \leq C k 2^{-k} \sum_{|\alpha|=m-1}\left\|D^{\alpha} A\right\|_{\mathrm{BMO}} .
$$

For the term III, by the following formula (see [20]):

$$
\begin{aligned}
& R_{m}(A ; x, y)-R_{m}(A ; x, z) \\
& \quad=\sum_{|\alpha|<m} \frac{1}{\beta !} R_{m-|\alpha|}\left(D^{\alpha} A ; z, y\right)(x-z)^{\alpha},
\end{aligned}
$$


and then together with Lemma 7, we have

$$
\begin{aligned}
I I I & \leq C \sum_{|\alpha|=m-1}\left\|D^{\alpha} A\right\|_{\mathrm{BMO}} \sum_{i=0}^{m-2}|x-y|^{-(m-1)+l}|y-u|^{m-1-i} \\
& \leq C \sum_{|\alpha|=m-1}\left\|D^{\alpha} A\right\|_{\mathrm{BMO}} k 2^{-k} .
\end{aligned}
$$

Thus for $\mu_{21}(x, u)$, by the size condition of $\widetilde{a}$, we get the following estimates:

$$
\begin{aligned}
& \left\|\mu_{21}(\cdot, u)\right\|_{L^{n /(n-\beta)}} \\
& \leq C \sum_{|\alpha|=m-1}\left\|D^{\alpha} A\right\|_{\mathrm{BMO}} \\
& \quad \times \sum_{k=2}^{\infty} k 2^{-k}\left[\int _ { 2 ^ { k + 1 } B | 2 ^ { k } B } \left(\frac{1}{|x|^{n-\beta}}\right.\right. \\
& \left.\left.\quad \times \int_{B}|\tilde{a}(y)| d y\right)^{n /(n-\beta)} d x\right]^{(n-\beta) / n} \\
& \leq C \sum_{|\alpha|=m-1}\left\|D^{\alpha} A\right\|_{\mathrm{BMO}} \sum_{k=2}^{\infty} k 2^{-k} \leq C \sum_{|\alpha|=m-1}\left\|D^{\alpha} A\right\|_{\mathrm{BMO}} .
\end{aligned}
$$

For $\mu_{22}(x, u)$, since $\Omega \in \operatorname{Lip}_{1}\left(S^{n-1}\right)$, we have the following estimates of $\left|K_{\alpha}(x-y)-K_{\alpha}(x-u)\right|$ :

$$
\begin{aligned}
\left|K_{\alpha}(x-y)-K_{\alpha}(x-u)\right| & \leq\left|\frac{\Omega(x-y)(x-y)^{\alpha}}{|x-y|^{m-1}}-\frac{\Omega(x-y)(x-u)^{\alpha}}{|x-y|^{m-1}}\right| \\
& +\left|\frac{\Omega(x-y)(x-u)^{\alpha}}{|x-y|^{m-1}}-\frac{\Omega(x-u)(x-u)^{\alpha}}{|x-y|^{m-1}}\right| \\
& +\left|\frac{\Omega(x-u)(x-u)^{\alpha}}{|x-y|^{m-1}}-\frac{\Omega(x-u)(x-u)^{\alpha}}{|x-u|^{m-1}}\right| \\
\leq & C \frac{|y-u|}{|x-y|} .
\end{aligned}
$$

Thus by the size condition of $\widetilde{a}$ and Lemma 7 , we have

$$
\begin{aligned}
& \left\|\mu_{22}(\cdot, u)\right\|_{L^{n /(n-\beta)}} \\
& \leq C \sum_{|\alpha|=m-1} \sum_{k=2}^{\infty}\left[\int_{2^{k+1} B \mid 2^{k} B}\right. \\
& \quad \times\left(\int_{B} \frac{|y-u|}{|x-y||x|^{n-\beta}}|\widetilde{a}(y)|\right. \\
& \left.\left.\quad \times\left|D^{\alpha}(\widetilde{A})(y)\right| d y\right)^{n /(n-\beta)} d x\right]^{(n-\beta) / n}
\end{aligned}
$$

$$
\begin{aligned}
& \leq C \sum_{|\alpha|=m-1}\left\|D^{\alpha} A\right\|_{\mathrm{BMO}} \\
& \quad \times \sum_{k=2}^{\infty}\left|2^{k} B\right|^{(\beta-n) / n}|B|^{-1}|B|\left|2^{k} B\right|^{(n-\beta) / n} 2^{-k} \\
& \leq C \sum_{|\alpha|=m-1}\left\|D^{\alpha} A\right\|_{\mathrm{BMO}} .
\end{aligned}
$$

Now we can deduce that $\left\|\mathscr{H}_{\Omega, A, \beta}^{m} \widetilde{a}\right\|_{L^{n /(n-\beta)}} \leq C$ is equivalent to $\left\|\mu_{23}(\cdot, u)\right\|_{L^{n /(n-\beta)}} \leq C$. By the vanishing condition of $\widetilde{a}$, we can easily get

$$
\begin{aligned}
& \int_{(4 B)^{c}}\left|\sum_{|\alpha|=m-1} \frac{1}{\alpha !} K_{\alpha}(x, u) \frac{1}{|x|^{n-\beta}} \int_{B} D^{\alpha} A(y) \tilde{a}(y) d y\right|^{n /(n-\beta)} \\
& \quad \times d x \leq C .
\end{aligned}
$$

Consequently, we have finished the proof of Theorem 6 .

Next we would like to show that $\mathscr{H}_{\Omega, A, \beta}^{m}$ is not bounded from $H^{1}$ to $L^{n /(n-\beta)}$ unless $\mathscr{H}_{\Omega, A, \beta}^{m} \equiv 0$. We have the following theorem.

Theorem 8. Let $m \geq 2,0 \leq \beta<n$, and $\Omega \in \operatorname{Lip}_{1}\left(S^{n-1}\right)$, and assume that $A$ has derivatives of order $m-1$ in $B M O\left(\mathbb{R}^{n}\right)$. Then the following two statements are equivalent:

(i) $\mathscr{H}_{\Omega, A, \beta}^{m}$ maps $H^{1}$ continuously into $L^{n /(n-\beta)}$,

(ii) $A$ is a polynomial of degree no more than $m-1$ or $\Omega \equiv$ 0 .

Remark 9. From Theorem 8, we can draw the conclusion that, when $m \geq 2, \mathscr{H}_{\Omega, A, \beta}^{m}$ is not bounded from $H^{1}$ to $L^{n /(n-\beta)}$ unless $\mathscr{H}_{\Omega, A, \beta}^{m} \equiv 0$.

Proof of Theorem 8. It is clear that (ii) $\Rightarrow$ (i) is obvious. We only need to prove (i) $\Rightarrow$ (ii).

Let $\tilde{a}$ be an $H^{1}$ atom supported on the ball $B=B(0, r)$, and denote $C_{\alpha}=(1 / \alpha !) \int_{B} D^{\alpha} A(y) \widetilde{a}(y) d y$ with $|\alpha|=m-1$. By Theorem 6 , for any $u \in 3 B \backslash 2 B$ and $N>8$ with $N \in Z^{+}$, we have

$$
\begin{aligned}
C & \geq \int_{(4 B)^{c}}\left|\frac{1}{|x|^{n-\beta}} \sum_{|\alpha|=m-1} C_{\alpha} \frac{\Omega(x-u)(x-u)^{\alpha}}{|x-u|^{m-1}}\right|^{n /(n-\beta)} d x \\
& \geq C_{1} \int_{(4 B)^{c}}\left|\frac{1}{|x-u|^{n-\beta}} \sum_{|\alpha|=m-1} C_{\alpha} \frac{\Omega(x-u)(x-u)^{\alpha}}{|x-u|^{m-1}}\right|^{n /(n-\beta)} d x
\end{aligned}
$$




$$
\begin{aligned}
& \geq C_{1} \int_{8 r \leq|x| \leq N r}\left|\frac{1}{|x|^{n-\beta}} \sum_{|\alpha|=m-1} C_{\alpha} \Omega(x) \frac{x^{\alpha}}{|x|^{m-1}}\right|^{n /(n-\beta)} d x \\
& =C_{1} \log \left(\frac{N}{8}\right) \int_{S^{n-1}}\left|\sum_{|\alpha|=m-1} C_{\alpha} \Omega\left(x^{\prime}\right)\left(x^{\prime}\right)^{\alpha}\right|^{n /(n-\beta)} d \sigma\left(x^{\prime}\right) .
\end{aligned}
$$

Let $N \rightarrow+\infty$, we know $\log (N / 8) \rightarrow+\infty$. Thus we have

$$
\int_{S^{n-1}}\left|\sum_{|\alpha|=m-1} C_{\alpha} \Omega\left(x^{\prime}\right)\left(x^{\prime}\right)^{\alpha}\right|^{n /(n-\beta)} d \sigma\left(x^{\prime}\right)=0 .
$$

From (44) we can deduce

$$
\sum_{|\alpha|=m-1} C_{\alpha} \Omega\left(x^{\prime}\right)\left(x^{\prime}\right)^{\alpha}=0
$$

If $\Omega \equiv 0,(45)$ is obviously true. Otherwise, we can easily obtain

$$
C_{\alpha}=\frac{1}{\alpha !} \int_{B} D^{\alpha} A(y) \tilde{a}(y) d y=0
$$

Since $\tilde{a}$ is arbitrary, $D^{\alpha} A$ must be a constant for each $\alpha$ with $|\alpha|=m-1$. So we can deduce that $A$ is a polynomial with degree no more than $m-1$.

Consequently, we have finished the proof of Theorem 8.

\section{Boundedness of $\mathscr{H}_{\Omega, A, \beta}^{m}$ from $H^{1}$ to $L^{n /(n-\beta), \infty}$}

In Section 2, we prove that, when $m \geq 2, \mathscr{H}_{\Omega, A, \beta}^{m}$ is not bounded from $H^{1}$ to $L^{n /(n-\beta)}$ unless $\mathscr{H}_{\Omega, A, \beta}^{m} \equiv 0$. In this section, we will prove that $\mathscr{H}_{\Omega, A, \beta}^{m}$ is bounded from $H^{1}$ to $L^{n /(n-\beta), \infty}$ with $m \geq 1$. Here $L^{n /(n-\beta), \infty}$ is defined by

$$
\|f\|_{L^{n /(n-\beta), \infty}}=\sup _{\lambda>0} \lambda \mid\left\{x \in \mathbb{R}^{n}:|f(x)|>\left.\lambda\right|^{(n-\beta) / n}<\infty .\right.
$$

Our results can be stated as follows.

Theorem 10. Suppose that $m \geq 2,0 \leq \beta<n$, and $\Omega \in$ $\operatorname{Lip}_{1}\left(S^{n-1}\right)$. If $A$ has derivatives of order $m-1$ in $B M O\left(\mathbb{R}^{n}\right)$, then there exists a constant $C$ independent of $f$ and $A$, such that

$$
\begin{gathered}
\left|\left\{x \in \mathbb{R}^{n}:\left|\mathscr{H}_{\Omega, A, \beta}^{m} f(x)\right|>\lambda\right\}\right|^{(n-\beta) / n} \\
\leq C \sum_{|\alpha|=m-1}\left\|D^{\alpha} A\right\|_{B M O} \frac{\|f\|_{H^{1}}}{\lambda}
\end{gathered}
$$

for any $\lambda>0$.

For the case $m=1$, we have the following.
Theorem 11. Let $0 \leq \beta<n, A \in B M O\left(\mathbb{R}^{n}\right)$, and $\Omega \in$ Lip $\left(S^{n-1}\right)$; then there exists a constant $C$ independent of $f$ and $A$, such that

$$
\begin{aligned}
\mid\{x & \left.\in \mathbb{R}^{n}:\left|\mathscr{H}_{\Omega, A, \beta}^{1} f(x)\right|>\lambda\right\}\left.\right|^{(n-\beta) / n} \\
& \leq C \sum_{|\alpha|=m-1}\left\|D^{\alpha} A\right\|_{B M O} \frac{\|f\|_{H^{1}}}{\lambda}
\end{aligned}
$$

for any $\lambda>0$.

Before the proof of Theorems 10 and 11, we need the following lemma.

Lemma 12. Let $\widetilde{\mathscr{H}}_{\beta}^{\alpha}$ be defined by

$$
\widetilde{\mathscr{H}}_{\beta}^{\alpha} h(x)=\frac{1}{|x|^{n-\beta}} \int_{|y|<|x|} h(y)(x-y)^{\alpha} \frac{\Omega(x-y)}{|x-y|^{m-1}} d y,
$$

where $0 \leq \beta<n, \Omega \in \operatorname{Lip}_{1}\left(S^{n-1}\right)$, and $|\alpha|=m-1$; then $\widetilde{\mathscr{H}}_{\beta}^{\alpha}$ is bounded from $L^{1}$ to $L^{n /(n-\beta), \infty}$.

Proof. For any $\lambda>0$, we have

$$
\begin{aligned}
& \lambda\left|\left\{x \in \mathbb{R}^{n}:\left|\widetilde{\mathscr{H}}_{\beta}^{\alpha} h(x)\right|>\lambda\right\}\right|^{(n-\beta) / n} \\
& =\lambda \mid\left\{x \in \mathbb{R}^{n}: \mid \frac{1}{|x|^{n-\beta}} \int_{|y|<|x|} h(y)(x-y)^{\alpha}\right. \\
& \left.\times \frac{\Omega(x-y)}{|x-y|^{m-1}} d y \mid>\lambda\right\}\left.\right|^{(n-\beta) / n} \\
& \leq \lambda\left|\left\{x \in \mathbb{R}^{n}:\left|\frac{1}{|x|^{n-\beta}} \int_{|y|<|x|}\right| h(y)\left|\frac{1}{|x-y|^{m-1-|\alpha|}} d y\right|>\lambda\right\}\right|^{(n-\beta) / n} \\
& \leq \lambda\left|\left\{x \in \mathbb{R}^{n}:\left|\frac{1}{|x|^{n-\beta}} \int_{|y|<|x|}\right| h(y)|d y|>\lambda\right\}\right|^{(n-\beta) / n} \\
& \leq \lambda\left|\left\{x \in \mathbb{R}^{n}:|x|<\frac{\|h\|_{L^{1}}^{1 /(n-\beta)}}{\lambda^{1 /(n-\beta)}}\right\}\right|^{(n-\beta) / n} \\
& =\lambda\left(\int_{S^{n-1}} \int_{0}^{\left(\|h\|_{L^{1}}^{1 /(n-\beta)}\right) /\left(\lambda^{1 /(n-\beta)}\right)} r^{n-1} d r d \sigma\left(x^{\prime}\right)\right)^{(n-\beta) / n} \\
& \leq C \lambda\left(\frac{\|h\|_{L^{1}}^{n /(n-\beta)}}{\lambda^{n /(n-\beta)}}\right)^{(n-\beta) / n}=C\|h\|_{L^{1}} .
\end{aligned}
$$

Proposition 13. By the proof of Lemma 12 with minor changes, one can draw the conclusion that $\mathscr{H}_{\Omega, \beta}$ is bounded from $L^{1}$ to $L^{n /(n-\beta), \infty}$ with $\Omega \in \operatorname{Lip}_{1}\left(S^{n-1}\right)$. 
Proof of Theorem 10. Before giving the proof of Theorem 10, we introduce some notations that are very useful in this section.

For multi-indices $\alpha=\left(\alpha_{1}, \ldots, \alpha_{n}\right), \beta=\left(\beta_{1}, \ldots, \beta_{n}\right)$, we denote $\alpha-\beta=\left(\alpha_{1}-\beta_{1}, \ldots, \alpha_{n}-\beta_{n}\right)$. Furthermore, $\beta<\alpha$ means that for each $i$, we have $\beta_{i}<\alpha_{i}$. Finally, we denote $C_{\alpha}^{\beta}=\prod_{j=1}^{m} C_{\alpha_{j}}^{\beta_{j}}$.

From [19] and by the atomic decomposition of $H^{1}$, it suffices to show

$$
\left|\left\{x \in \mathbb{R}^{n}:\left|\mathscr{H}_{\Omega, A, \beta}^{m} \widetilde{a}(x)\right|>\lambda\right\}\right|^{(n-\beta) / n} \leq \frac{C}{\lambda} \sum_{|\alpha|=m-1}\left\|D^{\alpha} A\right\|_{\mathrm{BMO}}
$$

for any $H^{1}$ atom $\widetilde{a}$, where $\widetilde{a}$ is defined in Section 2 . First we have the following decomposition:

$$
\begin{aligned}
\left|\left\{x \in \mathbb{R}^{n}: \mathscr{H}_{\Omega, A, \beta}^{m} \widetilde{a}(x)>\lambda\right\}\right|^{(n-\beta) / n} \\
\leq\left|\left\{x \in B(0,2 r): \mathscr{H}_{\Omega, A, \beta}^{m} \widetilde{a}(x)>\lambda\right\}\right|^{(n-\beta) / n} \\
\quad+\left|\left\{x \in \mathbb{R}^{n} \backslash B(0,2 r): \mathscr{H}_{\Omega, A, \beta}^{m} \widetilde{a}(x)>\lambda\right\}\right|^{(n-\beta) / n} \\
:=L_{1}+L_{2} .
\end{aligned}
$$

For the term $L_{1}$, choosing $B(0, r)=B$ and $B(0, k r)=k B$ with $k \in Z^{+}$, then by Proposition 2, Hölder inequality, and the size condition of $\widetilde{a}$, we have

$$
\begin{aligned}
& \lambda\left|\left\{x \in B(0,2 r): \mathscr{H}_{\Omega, A, \beta}^{m} \widetilde{a}(x)>\lambda\right\}\right|^{(n-\beta) / n} \\
& \leq C\left(\int_{B(0,2 r)}\left|\mathscr{H}_{\Omega, A, \beta}^{m} \widetilde{a}(x)\right|^{n /(n-\beta)} d x\right)^{(n-\beta) / n} \\
& \leq C|2 B|^{(n-\beta) / n-1 / q}\left(\int_{B(0,2 r)}\left|\mathscr{H}_{\Omega, A, \beta}^{m} \widetilde{a}(x)\right|^{q} d x\right)^{1 / q} \\
& \leq C|2 B|^{(n-\beta) / n-1 / q} \sum_{|\alpha|=m-1}\left\|D^{\alpha} A\right\|_{\mathrm{BMO}}\|\widetilde{a}\|_{L^{p}} \\
& \leq C \sum_{|\alpha|=m-1}\left\|D^{\alpha} A\right\|_{\mathrm{BMO}},
\end{aligned}
$$

where $1 / p-1 / q=\beta / n$ and $p>1$.

For the term $L_{2}$, as $x \in \mathbb{R}^{n} \backslash B(0,2 r)$, we have

$$
\{y:|y|<|x|\} \cap\{y:|y|<r\}=\{y:|y|<r\} .
$$

Then for a fixed $B=B(0, r)$, we set $A_{B}(x)=A(x)-$ $\sum_{|\alpha|=m-1}(1 / \alpha !)\left(D^{\alpha} A\right)_{B} x^{\alpha}$. It is easy to check $R_{m}(A ; x, y)=$ $R_{m}\left(A_{B} ; x, y\right)$.
Thus by the vanishing condition of $\widetilde{a}$ and the fact $\operatorname{supp}(\widetilde{a}) \subset B(0, r)$, we can decompose $\mathscr{H}_{\Omega, A, \beta}^{m} \widetilde{a}(x)$ as follows:

$$
\begin{aligned}
& \mathscr{H}_{\Omega, A, \beta}^{m} \tilde{a}(x) \\
& =\frac{1}{|x|^{n-\beta}} \int_{\mathbb{R}^{n}}\left\{R_{m}\left(A_{B} ; x, y\right) \frac{\Omega(x-y)}{|x-y|^{m-1}}\right. \\
& -\left[R_{m-1}\left(A_{B} ; x, 0\right)-\sum_{|\alpha|=m-1} \frac{1}{\alpha !} D^{\alpha} A_{B}(x) x^{\alpha}\right] \\
& \left.\times \frac{\Omega(x)}{|x|^{m-1}}\right\} \widetilde{a}(y) d y \\
& =-\frac{1}{|x|^{n-\beta}} \int_{\mathbb{R}^{n}}\left[R_{m-1}\left(A_{B} ; x, 0\right)-\sum_{|\alpha|=m-1} \frac{1}{\alpha !} D^{\alpha} A_{B}(x) x^{\alpha}\right] \\
& \times\left[\frac{\Omega(x)}{|x|^{m-1}}-\frac{\Omega(x-y)}{|x-y|^{m-1}}\right] \widetilde{a}(y) d y+\frac{1}{|x|^{n-\beta}} \\
& \times \int_{\mathbb{R}^{n}}\left[\sum_{|\alpha|<m-1} \frac{1}{\alpha !} D^{\alpha} A_{B}(y)(x-y)^{\alpha}\right. \\
& \left.-\sum_{|\alpha|<m-1} D^{\alpha} A_{B}(0) x^{\alpha}\right] \frac{\Omega(x-y)}{|x-y|^{m-1}} \widetilde{a}(y) d y+\frac{1}{|x|^{n-\beta}} \\
& \times \int_{\mathbb{R}^{n}}\left[\sum_{|\alpha|=m-1} \frac{1}{\alpha !} D^{\alpha} A_{B}(y) x^{\alpha}\right. \\
& \left.-\sum_{|\alpha|=m-1} \frac{1}{\alpha !} D^{\alpha} A_{B}(x) x^{\alpha}\right] \frac{\Omega(x-y)}{|x-y|^{m-1}} \widetilde{a}(y) d y \\
& +\frac{1}{|x|^{n-\beta}} \int_{\mathbb{R}^{n}} \sum_{|\alpha|=m-1} \frac{1}{\alpha !} D^{\alpha} A_{B}(y) \\
& \times \sum_{\gamma<\alpha} C_{\alpha}^{\gamma} x^{\gamma}(-y)^{\alpha-\gamma} \frac{\Omega(x-y)}{|x-y|^{m-1}} \widetilde{a}(y) d y \\
& :=I_{1}+I_{2}+I_{3}+I_{4} \text {. }
\end{aligned}
$$

Here we can simply denote each $I_{i}$ by

$$
I_{i}=\frac{1}{|x|^{n-\beta}} \int_{\mathbb{R}^{n}} K_{i}\left(A_{B} ; x, y\right) \tilde{a}(y) d y .
$$

For $K_{1}$, by the fact that $\Omega \in \operatorname{Lip}_{1}\left(S^{n-1}\right)$ and $|x-y| \sim|x|$, we have

$$
\begin{aligned}
& \left|\frac{\Omega(x)}{|x|^{m-1}}-\frac{\Omega(x-y)}{|x-y|^{m-1}}\right| \leq\left|\frac{\Omega(x)}{|x|^{m-1}}-\frac{\Omega(x-y)}{|x|^{m-1}}\right| \\
& +\left|\frac{\Omega(x-y)}{|x|^{m-1}}-\frac{\Omega(x-y)}{|x-y|^{m-1}}\right|
\end{aligned}
$$




$$
\begin{aligned}
= & \left|\frac{\Omega((x /|x|)(|x| /|x-y|))}{|x|^{m-1}}-\frac{\Omega((x-y) /|x-y|)}{|x|^{m-1}}\right| \\
& +\left|\frac{\Omega(x-y)}{|x|^{m-1}}-\frac{\Omega(x-y)}{|x-y|^{m-1}}\right| \leq C \frac{|y|}{|x|^{m}} .
\end{aligned}
$$

As $|x|>r$ for $x \in \mathbb{R}^{n} \backslash 2 B$, Lemma 7 in this paper and Lemma 2.2 in [8] tell us that

$$
\begin{aligned}
& \left|K_{1}\left(A_{B} ; x, y\right)\right| \\
& \leq C|x|^{m-1}\left[\sum_{|\alpha|=m-1}\left(\frac{1}{|\widetilde{Q}|} \int_{\widetilde{Q}}\left|D^{\alpha} A(z)-\left(D^{\alpha} A\right)_{B}\right|^{q} d z\right)^{q}\right. \\
& \left.\quad+\sum_{|\alpha|=m-1}\left|D^{\alpha} A(x)-\left(D^{\alpha} A\right)_{B}\right|\right] \frac{|y|}{|x|^{m}} \\
& \leq C \frac{|y|}{|x|}\left(\sum_{|\alpha|=m-1}\left\|D^{\alpha} A\right\|_{\mathrm{BMO}}\left(C_{1}+\log \frac{|x|}{r}\right)\right. \\
& \left.\quad+\sum_{|\alpha|=m-1}\left|D^{\alpha} A(x)-\left(D^{\alpha} A\right)_{B}\right|\right),
\end{aligned}
$$

where $C_{1}$ is a constant only depending on $n$, and $\widetilde{\mathrm{Q}}$ is a cube centered at $x$ and having diameter $5 \sqrt{n}|x|$.

Thus we obtain

$$
\begin{aligned}
& \lambda\left|\left\{x \in \mathbb{R}^{n} \backslash B(0,2 r):\left|I_{1}\right|>\lambda\right\}\right|^{(n-\beta) / n} \\
& \leq\left(\int_{\mathbb{R}^{n} \backslash B(0,2 r)}\left|I_{1}\right|^{n /(n-\beta)} d x\right)^{(n-\beta) / n} \\
& \leq C\left(\int_{\mathbb{R}^{n} \backslash B(0,2 r)}|x|^{(\beta-n)(n /(n-\beta))}\right. \\
& \quad \times\left(\int _ { \mathbb { R } ^ { n } } \frac { | y | } { | x | } \left(\sum_{|\alpha|=m-1}\left\|D^{\alpha} A\right\|_{\mathrm{BMO}}\left(C_{1}+\log \frac{|x|}{r}\right)\right.\right. \\
& \left.\quad+\sum_{|\alpha|=m-1}\left(D^{\alpha} A(x)-\left(D^{\alpha} A\right)_{B}\right)\right) \\
& \leq C\left(\sum_{k=1}^{+\infty} \int_{2^{k+1} B \mid 2^{k} B}|x|^{-n}|B|^{1 /(n-\beta)}|x|^{n /(\beta-n)}\right. \\
& \quad \times\left(\sum_{|\alpha|=m-1}\left\|D^{\alpha} A\right\|_{\mathrm{BMO}}\left(C_{1}+\log \frac{|x|}{r}\right)\right. \\
& \left.\left.\quad+\sum_{|\alpha|=m-1}\left(D^{\alpha} A(x)-\left(D^{\alpha} A\right)_{B}\right)\right)^{(n-\beta) / n} d x\right)^{n /(n-\beta)} d(n-\beta) / n
\end{aligned}
$$

$$
\begin{aligned}
& \leq C\left(\sum_{k=1}^{+\infty}|B|^{1 /(n-\beta)}\left|2^{k} B\right|^{(1 /(\beta-n))-1} \int_{2^{k+1} B \mid 2^{k} B}\right. \\
& \times\left(\sum_{|\alpha|=m-1}\left\|D^{\alpha} A\right\|_{\mathrm{BMO}}\left(C_{1}+\log \frac{|x|}{r}\right)\right. \\
& \left.\left.+\sum_{|\alpha|=m-1}\left(D^{\alpha} A(x)-\left(D^{\alpha} A\right)_{B}\right)\right)^{n /(n-\beta)} d x\right)^{(n-\beta) / n} \\
& \leq C\left(\sum_{|\alpha|=m-1}\left\|D^{\alpha} A\right\|_{\mathrm{BMO}}^{n /(n-\beta)} \sum_{k=1}^{+\infty}\left(C_{1}+\log \frac{\left|2^{k+1} r\right|}{r}\right)^{n /(n-\beta)}\right. \\
& \left.\times|B|^{1 /(n-\beta)}\left|2^{k+1} B\right|^{(1 /(\beta-n))-1}\left|2^{k+1} B\right|\right)^{(n-\beta) / n} \\
& +\left(\sum_{k=1}^{+\infty}|B|^{1 /(n-\beta)}\left|2^{k} B\right|^{(1 /(n-\beta))-1} \int_{2^{k+1} B \mid 2^{k} B}\right. \\
& \left.\times \sum_{|\alpha|=m-1}\left(D^{\alpha} A(x)-\left(D^{\alpha} A\right)_{B}\right)^{n /(n-\beta)} d x\right)^{(n-\beta) / n} \\
& \leq C\left(\sum_{|\alpha|=m-1}\left\|D^{\alpha} A\right\|_{\mathrm{BMO}}^{n /(n-\beta)} \sum_{k=1}^{\infty}\left(k+C_{1}\right)^{n /(n-\beta)}|B|^{1 /(n-\beta)}\right. \\
& \left.\times\left|2^{k+1} B\right|^{(1 /(\beta-n))-1}\left|2^{k+1} B\right|\right)^{(n-\beta) / n} \\
& \leq C \sum_{|\alpha|=m-1}\left\|D^{\alpha} A\right\|_{\text {BMO }} .
\end{aligned}
$$

Next we will give the estimates of $I_{2}$. First by a cumbersome but straightforward computation, we have

$$
\left|K_{2}\left(A_{B}, x, y\right)\right| \leq \sum_{k=0}^{m-2} \sum_{|\alpha|=k}\left|R_{m-k-1}\left(D^{\alpha} A_{B} ; 0, y\right)\right||x|^{-m+k+1} .
$$

Also noting the fact that

$$
\begin{aligned}
& \left|R_{m-k-1}\left(D^{\alpha} A_{B} ; 0, y\right)\right| \\
& \leq C|y|^{m-k-1} \\
& \quad \times \sum_{|\alpha|=m-1}\left(\frac{1}{\left|\widetilde{Q}_{0}\right|} \int_{\widetilde{Q}_{0}}\left|D^{\alpha} A(z)-\left(D^{\alpha} A\right)_{B}\right|^{q} d z\right)^{1 / q} \\
& \leq C r^{m-k-1} \sum_{|\alpha|=m-1}\left\|D^{\alpha} A\right\|_{\text {BMO }},
\end{aligned}
$$

where $q>n$, and $\widetilde{Q}_{0}$ is a cube centered at 0 and having diameter $5 \sqrt{n}|y|$.

Thus we obtain

$$
\left|K_{2}\left(A_{B}, x, y\right)\right| \leq C \sum_{|\alpha|=m-1}\left\|D^{\alpha} A\right\|_{\text {BMO }} \sum_{k=0}^{m-2} r^{m-k-1}|x|^{-m+k+1} \text {. }
$$


So we have the following estimates of $I_{2}$ :

$$
\begin{aligned}
I_{2} & \leq C \frac{1}{|x|^{n-\beta}} \int_{\mathbb{R}^{n}} \sum_{k=0}^{m-2} r^{m-k-1}|x|^{-m+k+1}|\widetilde{a}(y)| d y \\
& =C \sum_{k=0}^{m-2} r^{m-k-1}|x|^{-m+k+1-n+\beta} \int_{B}|\widetilde{a}(y)| d y \\
& \leq C \sum_{k=0}^{m-2} r^{m-k-1}|x|^{-m+k+1-n+\beta} .
\end{aligned}
$$

Now we get

$$
\begin{aligned}
& \lambda\left|\left\{x \in \mathbb{R}^{n} \backslash B(0,2 r):\left|I_{2}\right|>\lambda\right\}\right|^{(n-\beta) / n} \\
& \leq C \sum_{|\alpha|=m-1}\left\|D^{\alpha} A\right\|_{\mathrm{BMO}}\left(\int_{\mathbb{R}^{n} \backslash B(0,2 r)}\left|I_{2}\right|^{n /(n-\beta)} d x\right)^{(n-\beta) / n} \\
& \leq C \sum_{|\alpha|=m-1}\left\|D^{\alpha} A\right\|_{\mathrm{BMO}} \\
& \quad \times\left(\sum_{k=0}^{m-2} r^{(m-k-1)(n /(n-\beta))}\right. \\
& \left.\quad \times \sum_{j=1}^{+\infty} \int_{2^{j+1} B \backslash 2^{j} B}|x|^{(n(1+k-m) /(n-\beta))-n} d x\right)^{(n-\beta) / n}
\end{aligned}
$$$$
\leq C \sum_{|\alpha|=m-1}\left\|D^{\alpha} A\right\|_{\mathrm{BMO}}
$$

$$
\begin{aligned}
& \times\left(\sum_{k=0}^{m-2}|B|^{(m-k-1) /(n-\beta)}\right. \\
& \left.\quad \times \sum_{j=1}^{\infty}\left|2^{j+1} B\right|^{((1+k-m) /(n-\beta))-1}\left|2^{j+1} B\right|\right)^{(n-\beta) / n}
\end{aligned}
$$

$$
\begin{aligned}
& \leq C \sum_{|\alpha|=m-1}\left\|D^{\alpha} A\right\|_{\mathrm{BMO}} \\
& \times\left(\sum_{k=0}^{m-2}|B|^{((m-k-1) /(n-\beta))+((1+k-m) /(n-\beta))-1+1}\right. \\
& \left.\quad \times \sum_{j=1}^{\infty} 2^{j n(((1+k-m) /(n-\beta))-1+1)}\right)^{(n-\beta) / n}
\end{aligned}
$$$$
\leq C \sum_{|\alpha|=m-1}\left\|D^{\alpha} A\right\|_{\mathrm{BMO}} .
$$

For $I_{3}$, by the vanishing condition of $\widetilde{a}(y)$, we can split $I_{3}$ as follows:

$$
\begin{aligned}
& I_{3}=\frac{1}{|x|^{n-\beta}} \int_{\mathbb{R}^{n}}\left[\sum_{|\alpha|=m-1} \frac{1}{\alpha !} D^{\alpha} A_{B}(y) x^{\alpha}\right. \\
& \left.-\sum_{|\alpha|=m-1} \frac{1}{\alpha !} D^{\alpha} A_{B}(x) x^{\alpha}\right] \\
& \times \frac{\Omega(x-y)}{|x-y|^{m-1}} \widetilde{a}(y) d y \\
& \leq \frac{1}{|x|^{n-\beta}} \sum_{|\alpha|=m-1} \frac{1}{\alpha !} \int_{\mathbb{R}^{n}}\left[D^{\alpha} A(y)-\left(D^{\alpha} A\right)_{B}\right] \\
& \times(x-y)^{\alpha} \frac{\Omega(x-y)}{|x-y|^{m-1}} \widetilde{a}(y) d y \\
& +\frac{1}{|x|^{n-\beta}} \sum_{|\alpha|=m-1} \frac{1}{\alpha !} \int_{\mathbb{R}^{n}} D^{\alpha} A_{B}(y) \\
& \times \sum_{\gamma<\alpha} C_{\alpha}^{\gamma}(x-y)^{\gamma} y^{\alpha-\gamma} \\
& \times \frac{\Omega(x-y)}{|x-y|^{m-1}} \widetilde{a}(y) d y \\
& +\frac{1}{|x|^{n-\beta}} \sum_{|\alpha|=m-1} \frac{1}{\alpha !} \int_{\mathbb{R}^{n}} x^{\alpha}\left[D^{\alpha} A(x)-\left(D^{\alpha} A\right)_{B}\right] \\
& \times \frac{\Omega(x-y)}{|x-y|^{m-1}} \widetilde{a}(y) d y \\
& =I_{31}+I_{32}+I_{33} .
\end{aligned}
$$

For the term $I_{31}$, by Lemma 12 and the size condition of $\widetilde{a}$, we obtain

$$
\begin{aligned}
\lambda \mid\{x \in & \left.\mathbb{R}^{n} \backslash B(0,2 r):\left|I_{31}\right|>\lambda\right\} \mid \\
& \leq \sum_{|\alpha|=m-1}\left\|\left(D^{\alpha} A-\left(D^{\alpha} A\right)_{B}\right) \tilde{a}\right\|_{L^{1}} \\
& \leq \sum_{|\alpha|=m-1}\left\|D^{\alpha} A\right\|_{\mathrm{BMO}} .
\end{aligned}
$$

For the term $I_{32}$, by the vanishing condition of $\widetilde{a}$ and the fact $\Omega \in \operatorname{Lip}_{1}\left(S^{n-1}\right)$, we have

$$
\begin{aligned}
I_{32}=\frac{1}{|x|^{n-\beta}} \sum_{|\alpha|=m-1} \frac{1}{\alpha !} \int_{\mathbb{R}^{n}} & {\left[D^{\alpha} A_{B}(y)-\left(D^{\alpha} A\right)_{B}\right] } \\
& \times \sum_{|\gamma|<\alpha} C_{\alpha}^{\gamma}(x-y)^{\gamma} y^{\alpha-\gamma} \\
& \times \frac{\Omega(x-y)}{|x-y|^{m-1}} \times \widetilde{a}(y) d y
\end{aligned}
$$




$$
\begin{aligned}
& \leq C \frac{1}{|x|^{n-\beta}} \sum_{|\alpha|=m-1} \frac{1}{\alpha !} \int_{\mathbb{R}^{n}}\left|D^{\alpha} A_{B}(y)-\left(D^{\alpha} A\right)_{B}\right| \\
& \times \sum_{|\gamma|<m-1} r^{m-1-|\gamma|}|x-y|^{1-m+|\gamma|} \\
& \times|\widetilde{a}(y)| d y \\
& \leq C \sum_{|\gamma|<m-1} \frac{1}{|x|^{n-\beta}}|B|^{(m-1-|\gamma|) / n} \\
& \times \int_{\mathbb{R}^{n}} \sum_{|\alpha|=m-1}\left|D^{\alpha} A_{B}(y)-\left(D^{\alpha} A\right)_{B}\right| \\
& \times|x-y|^{1-m+|\gamma|}|\widetilde{a}(y)| d y .
\end{aligned}
$$

Thus we get

$$
\begin{aligned}
& \lambda\left|\left\{x \in \mathbb{R}^{n} \backslash B(0,2 r):\left|I_{32}\right|>\lambda\right\}\right|^{(n-\beta) / n} \\
& \leq\left(\int_{\mathbb{R}^{n} \backslash B(0,2 r)}\left|I_{32}\right|^{n /(n-\beta)} d x\right)^{(n-\beta) / n} \\
& \leq C\left(\sum_{|\gamma|<m-1}|B|^{((m-1-|\gamma|) / n)(n /(n-\beta))} \sum_{k=1}^{\infty} \int_{2^{k+1} B \backslash 2^{k} B}\right. \\
& \times\left(\frac{1}{|x|^{n-\beta}} \int_{\mathbb{R}^{n}} \sum_{|\alpha|=m-1}\left|D^{\alpha} A_{B}(y)-\left(D^{\alpha} A\right)_{B}\right|\right. \\
& \left.\left.\times|x-y|^{1-m+|\gamma|}|\tilde{a}(y)| d y\right)^{n /(n-\beta)} d x\right)^{(n-\beta) / n} \\
& \leq C\left(\sum_{|\gamma|<m-1}|B|^{(m-1-|\gamma|) /(n-\beta)} \sum_{k=1}^{\infty}\left|2^{k} B\right|^{(1-m+|\gamma|+\beta-n) /(n-\beta)}\left|2^{k} B\right|\right. \\
& \left.\times\left(\int_{B_{|\alpha|=m-1}}\left|D^{\alpha} A_{B}(y)-\left(D^{\alpha} A\right)_{B}\right||\tilde{a}(y)| d y\right)^{n /(n-\beta)}\right)^{(n-\beta) / n} \\
& \leq C \sum_{|\alpha|=m-1}\left\|D^{\alpha} A\right\|_{\mathrm{BMO}}\left(\sum_{|\gamma|<m-1}|B|^{(m-1-|\gamma|) /(n-\beta)}|B|^{(1-m+|\gamma|+\beta-n) /(n-\beta)}\right. \\
& \left.\times|B|^{1-1+1} \sum_{k=1}^{\infty} k 2^{k n((1-m+|\gamma|+\beta-n) /(n-\beta))}\right)^{(n-\beta) / n}
\end{aligned}
$$$$
\leq C \sum_{|\alpha|=m-1}\left\|D^{\alpha} A\right\|_{\mathrm{BMO}}
$$

For the term $I_{33}$, by the vanishing condition of $\tilde{a}, \Omega \in$ $\operatorname{Lip}_{1}\left(S^{n-1}\right)$, and (58), we have

$$
I_{33}=\frac{1}{|x|^{n-\beta}} \sum_{|\alpha|=m-1} \frac{1}{\alpha !} x^{\alpha}\left[D^{\alpha} A(x)-\left(D^{\alpha} A\right)_{B}\right]
$$

$$
\begin{aligned}
& \times \int_{\mathbb{R}^{n}}\left(\frac{\Omega(x-y)}{|x-y|^{m-1}}-\frac{\Omega(x)}{|x|^{m-1}}\right) \tilde{a}(y) d y \\
& \leq \frac{1}{|x|^{n-\beta}} \sum_{|\alpha|=m-1} \frac{1}{\alpha !} x^{\alpha}\left[D^{\alpha} A(x)-\left(D^{\alpha} A\right)_{B}\right] \\
& +\frac{1}{|x|^{n-\beta}} \sum_{|\alpha|=m-1} \frac{1}{\alpha !} x^{\alpha}\left[D^{\alpha} A(x)-\left(D^{\alpha} A\right)_{B}\right] \\
& \quad \times \int_{\mathbb{R}^{n}}\left|\frac{\Omega(x)}{|x-y|^{m-1}}-\frac{\Omega(x)}{|x-y|^{m-1}}\right||\tilde{a}(y)| d y \\
& \leq C \frac{1}{|x|^{n-\beta}} \sum_{|\alpha|=m-1} \frac{1}{|x|^{m-1}}|x|^{m-1}\left|D^{\alpha} A(x)-\left(D^{\alpha} A\right)_{B}\right| \\
& \times \int_{B} \frac{|y|}{|x-y|^{m}|\tilde{a}(y)| d y .}
\end{aligned}
$$

Thus by Lemma 2.2 in [8], we obtain

$$
\begin{aligned}
& \lambda\left|\left\{x \in \mathbb{R}^{n} \backslash B(0,2 r):\left|I_{33}\right|>\lambda\right\}\right|^{(n-\beta) / n} \\
& \leq\left(\int_{\mathbb{R}^{n} \backslash B(0,2 r)}\left|I_{33}\right|^{n /(n-\beta)} d x\right)^{(n-\beta) / n} \\
& \leq C\left(\int_{\mathbb{R}^{n} \backslash B(0,2 r)}\right. \\
& \quad \times\left.\left|\frac{1}{|x|^{n-\beta}} \sum_{|\alpha|=m-1} \frac{1}{\alpha !}\right| x\right|^{m-1}\left|D^{\alpha} A(x)-\left(D^{\alpha} A\right)_{B}\right| \\
& \left.\quad \times\left.\int_{B} \frac{|y|}{|x-y|^{m}}|\widetilde{a}(y)| d y\right|^{n /(n-\beta)} d x\right)^{(n-\beta) / n} \\
& \leq C\left(\sum_{k=1}^{\infty} \int_{2^{k+1} \backslash 2^{k} B}|x|^{(\beta-n-1)(n /(n-\beta))}|B|^{1 /(n-\beta)}\right. \\
& \left.\leq C \sum_{|\alpha|=m-1} \sum_{|\alpha|=m-1}\left|D^{\alpha} A \|_{B M O}^{\alpha} A(x)-\left(D^{\alpha} A\right)_{B}\right|^{n /(n-\beta)} d x\right)^{(n-\beta) / n}
\end{aligned}
$$

$$
\begin{gathered}
\times\left(\sum_{k=1}^{\infty}(k+1)\left|2^{k} B\right|^{(\beta-n-1) /(n-\beta)}\left|2^{k} B\right|\right. \\
\left.\times|B|^{1 /(n-\beta)}\right)^{(n-\beta) / n}
\end{gathered}
$$




$$
\leq C \sum_{|\alpha|=m-1}\left\|D^{\alpha} A\right\|_{\text {BMO }}
$$

Finally, we will give the estimates of $I_{4}$. By a similar argument as in the estimates of $I_{32}$, we can easily get

$$
\lambda\left|\left\{x \in \mathbb{R}^{n} \backslash B(0,2 r):\left|I_{4}\right|>\lambda\right\}\right|^{(n-\beta) / n} \leq C \sum_{|\alpha|=m-1}\left\|D^{\alpha} A\right\|_{\mathrm{BMO}} .
$$

Combining the estimates of $I_{1}, I_{2}, I_{3}$, and $I_{4}$, we finish the proof of Theorem 10.

Proof of Theorem 11. Theorem 11 was proved in [7] in the case $\beta=0$ and $\Omega \equiv 1$. For the case $0 \leq \beta<n$ and $\Omega \equiv 1$, we can easily prove Theorem 11 by the proof of Theorem 5.3 in [7] with minor changes. Then for the case $0 \leq \beta<n$ and $\Omega \in$ $\operatorname{Lip}_{1}\left(S^{n-1}\right)$, by the main idea used in the proof of Theorem 10, we can prove Theorem 11 easily and we omit the details here.

\section{Acknowledgments}

The authors would like to express their gratitude to the referee for his/her valuable suggestions. This work was partially supported by National Natural Science Foundation of China under Grants nos. 10931001, 11226104, and 11226108, Natural Science Foundation of Jiangxi Province under Grants no. 20114BAB211007, and the Science Foundation of Jiangxi Education Department under Grants no. GJJ13703. This work was also supported by the Key Laboratory of Mathematics and Complex System (Beijing Normal University), Ministry of Education, China.

\section{References}

[1] G. H. Hardy, "Note on a theorem of Hilbert," Mathematische Zeitschrift, vol. 6, no. 3-4, pp. 314-317, 1920.

[2] A. Bényi and C. Oh, "Best constants for certain multilinear integral operators," Journal of Inequalities and Applications, vol. 2006, Article ID 28582, 12 pages, 2006.

[3] A. Kufner and L.-E. Persson, Weighted Inequalities of Hardy Type, World Scientific, Singapore, 2003.

[4] M. Christ and L. Grafakos, "Best constants for two nonconvolution inequalities," Proceedings of the American Mathematical Society, vol. 123, no. 6, pp. 1687-1693, 1995.

[5] Z.-w. Fu, Z.-g. Liu, S.-z. Lu, and H.-b. Wang, "Characterization for commutators of $n$-dimensional fractional Hardy operators," Science in China A, vol. 50, no. 10, pp. 1418-1426, 2007.

[6] Z. Fu, S. Lu, and F. Y. Zhao, "Commutators of $n$-dimensional rough Hardy operators," Science China Mathematics, vol. 54, no. 1, pp. 95-104, 2011.

[7] F. Zhao, Z. Fu, and S. Z. Lu, "Endpoint estimates for $n$ dimensional Hardy operators and their commutators," Science China Mathematics, vol. 55, no. 10, pp. 1977-1990, 2012.

[8] J. Cohen, "A sharp estimate for a multilinear singular integral in $\mathbb{R}^{n}$," Indiana University Mathematics Journal, vol. 30, no. 5, pp. 693-702, 1981.
[9] J. Cohen and J. Gosselin, "A BMO estimate for multilinear singular integrals," Illinois Journal of Mathematics, vol. 30, no. 3, pp. 445-464, 1986.

[10] W. Chen and G. Hu, "Weak type $H^{1}, L^{1}$ estimate for a multilinear singular integral operator," Advances in Mathematics, vol. 30, no. 1, pp. 63-69, 2001.

[11] S. Lu and Q. Wu, "Endpoint estimates for certain commutators of fractional and singular integrals," Proceedings of the American Mathematical Society, vol. 131, no. 2, pp. 467-477, 2003.

[12] D. Y. Yan, Some problems on multilinear singular integral operators and multilinear oscillatory singular integral operators [Ph.D. thesis], Beijing Normal University, Beijing, China, 2001.

[13] C. Wang and Z. Zhang, "A new proof of Wu's theorem on vortex sheets," Science China Mathematics, vol. 55, no. 7, pp. 1449-1462, 2012.

[14] S. J. Wu, "Mathematical analysis of vortex sheets," Communications on Pure and Applied Mathematics, vol. 59, no. 8, pp. 10651206, 2006.

[15] Q. Wu and D. C. Yang, "On fractional multilinear singular integrals," Mathematische Nachrichten, vol. 239, pp. 215-235, 2002.

[16] J. García-Cuerva and J. L. R. de Francia, Weighted Norm Inequalities and Related Topics, vol. 116, North-Holland, Amsterdam, The Netherland, 1985.

[17] B. Muckenhoupt and R. L. Wheeden, "Weighted bounded mean oscillation and the Hilbert transform," Studia Mathematica, vol. 54, no. 3, pp. 221-237, 1976.

[18] S. Z. Lu and F. Y. Zhao, "CBMO estimates for multilinear Hardy operators," Acta Mathematica Sinica, vol. 26, no. 7, pp. 12451254, 2010.

[19] D. Yang and Y. Zhou, "A boundedness criterion via atoms for linear operators in Hardy spaces," Constructive Approximation, vol. 29, no. 2, pp. 207-218, 2009.

[20] B. Bajsanski and R. Coifman, "On singular integrals," in Proceedings of Symposia in Pure Mathematics, vol. 10, pp. 1-17, American Mathematical Society, Chicago, Ill, USA, 1967. 


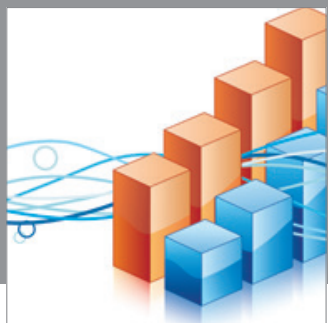

Advances in

Operations Research

mansans

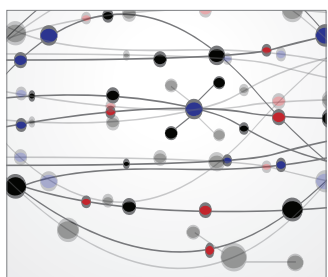

The Scientific World Journal
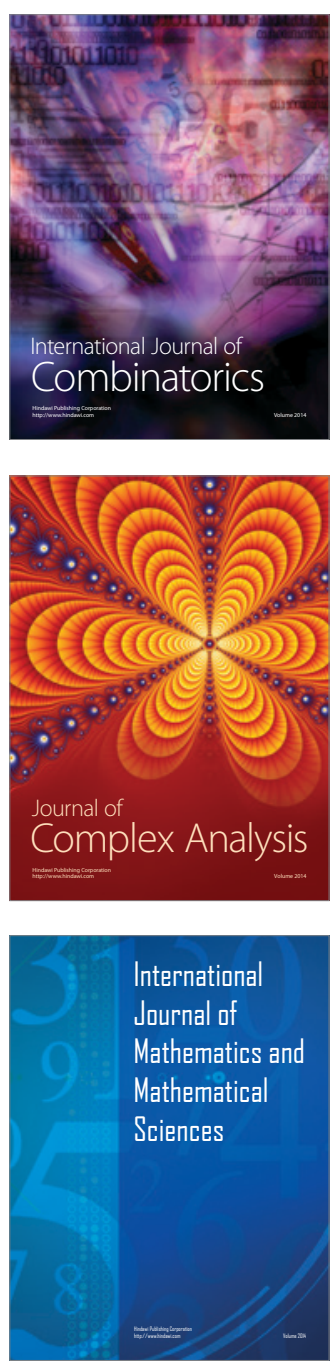
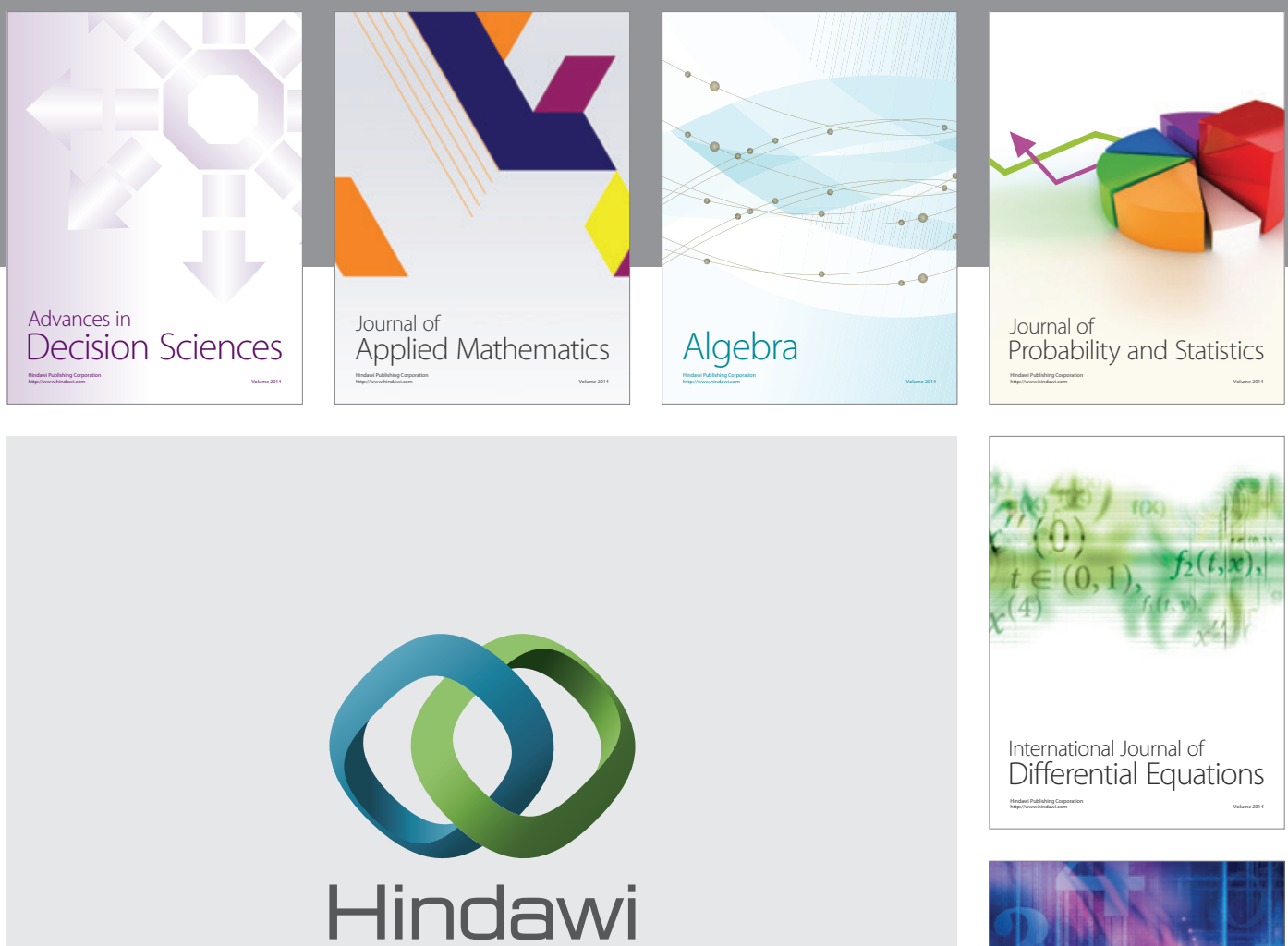

Submit your manuscripts at http://www.hindawi.com
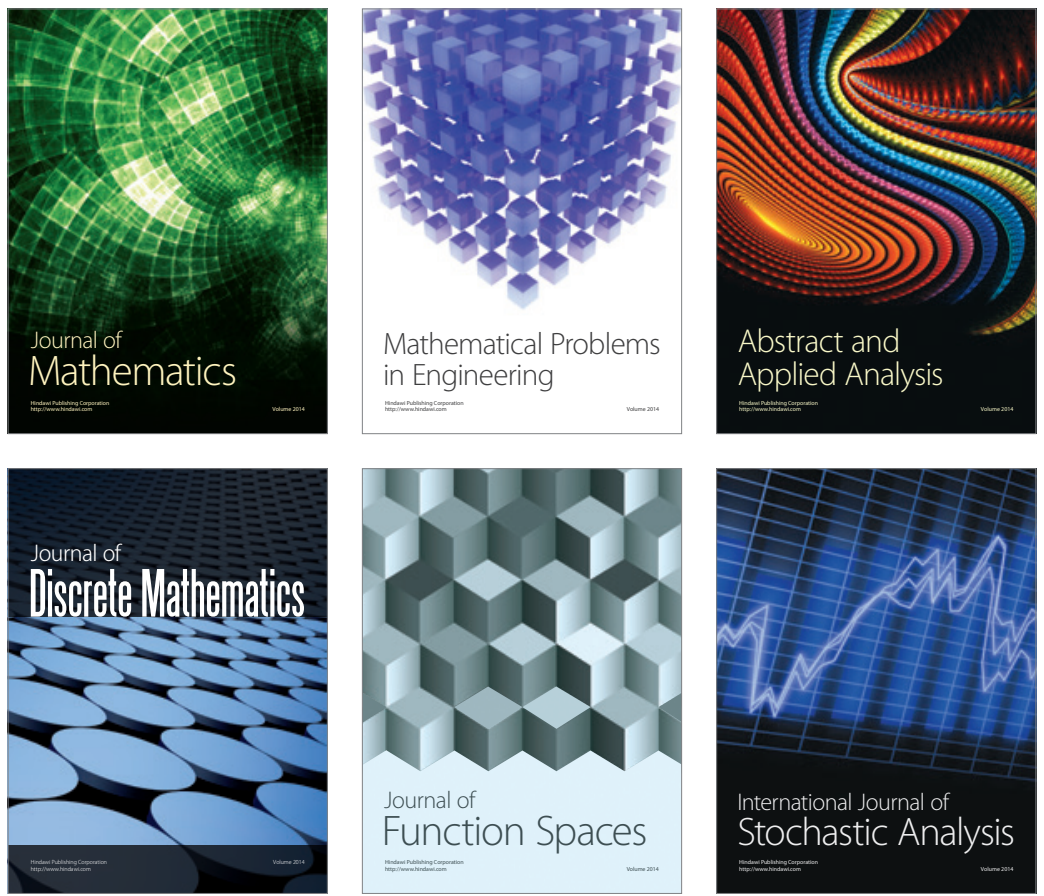

Journal of

Function Spaces

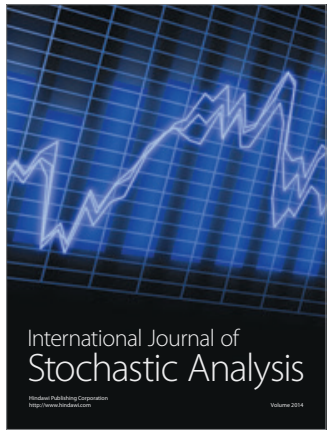

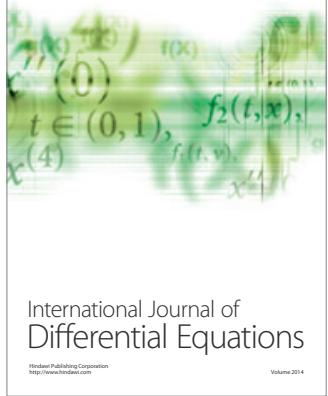
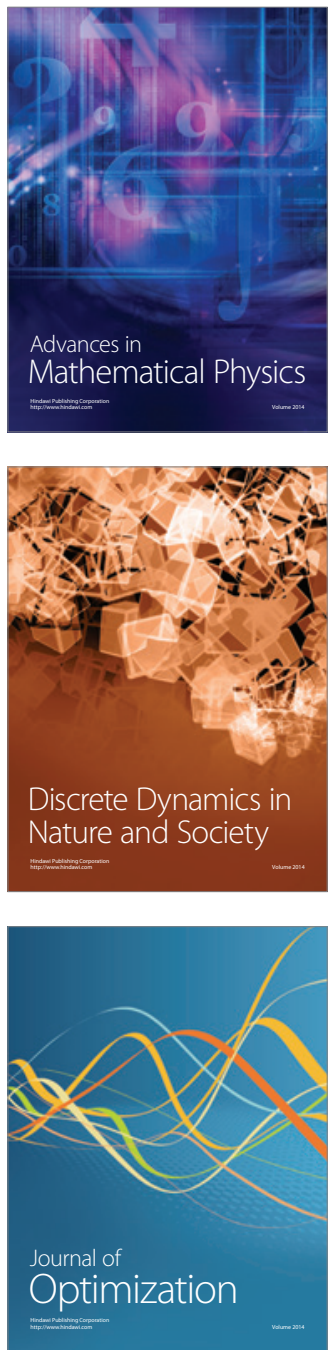